ARTICLE

\title{
Structure of the mini-RNA-guided endonuclease CRISPR-Cas12j3
}

\author{
Arturo Carabias ${ }^{1}$, Anders Fuglsang (10 1, Piero Temperini ${ }^{1}$, Tillmann Pape (10 1,2, Nicholas Sofos ${ }^{1}$, \\ Stefano Stella (1) ${ }^{1,4}$, Simon Erlendsson ${ }^{1,3} \&$ Guillermo Montoya (i) ${ }^{1 凶}$
}

CRISPR-Cas12j is a recently identified family of miniaturized RNA-guided endonucleases from phages. These ribonucleoproteins provide a compact scaffold gathering all key activities of a genome editing tool. We provide the first structural insight into the Cas12j family by determining the cryoEM structure of Cas12j3/R-loop complex after DNA cleavage. The structure reveals the machinery for PAM recognition, hybrid assembly and DNA cleavage. The crRNA-DNA hybrid is directed to the stop domain that splits the hybrid, guiding the T-strand towards the catalytic site. The conserved RuvC insertion is anchored in the stop domain and interacts along the phosphate backbone of the crRNA in the hybrid. The assembly of a hybrid longer than 12-nt activates catalysis through key functional residues in the RuvC insertion. Our findings suggest why Cas12j unleashes unspecific ssDNA degradation after activation. A site-directed mutagenesis analysis supports the DNA cutting mechanism, providing new avenues to redesign CRISPR-Cas12j nucleases for genome editing.

\footnotetext{
${ }^{1}$ Structural Molecular Biology Group, Novo Nordisk Foundation Centre for Protein Research, Faculty of Health and Medical Sciences University of Copenhagen, Copenhagen, Denmark. ${ }^{2}$ Core Facility for Integrated Microscopy (CFIM), Faculty of Health and Medical Sciences, University of Copenhagen, Copenhagen, Denmark. ${ }^{3}$ Structural Studies Division, MRC Laboratory of Molecular Biology, Cambridge, UK. ${ }^{4}$ Present address: Twelve Bio ApS,

Copenhagen, Denmark. ${ }^{凶}$ email: guillermo.montoya@cpr.ku.dk
} 
C ompetition between microbes and their invaders has driven the evolution of defence systems preventing the attack of mobile genetic elements. Among them, CRISPR constitutes a type of adaptive immunity achieved by CRISPRassociated nucleases (Cas) and CRISPR RNAs (crRNAs) that assemble effector RNPs, which are guided by the crRNA to cleave complementary DNA (or RNA) for interference ${ }^{1-3}$. Depending on the type of RNP effector nucleases, CRISPR-Cas systems can be categorized into two classes (1 and 2), which are further subdivided into six types (types I through VI). Class 2 systems form an RNP complex between the multidomain effector Cas protein and a CRISPR RNA (crRNA) that contains the necessary information to target a specific nucleic acid sequence ${ }^{2,4,5}$.

Although ubiquitously diversified among prokaryotes, CRISPR systems were also identified in bacteriophages ${ }^{6}$. Recently, a new Class 2 family of CRISPR nucleases named Cas12j, also known as Cas $\Phi$, was found in the biggiephage clade of phages ${ }^{7}$. Cas $12 j$ proteins share low sequence identity with other CRISPR nucleases and display sequence homology only in their RuvC domain with Class2 type V members. They generate a staggered DNA doublestrand break (DSB) and unleash unspecific ssDNA cleavage after activation with an ssDNA complementary to the crRNA, as other members of Class 2 type $\mathrm{V}^{7,8}$. In addition, the RuvC catalytic site of Cas12j1 and 2 also processes the precursor crRNA (precrRNA $)^{7}$. This family of endonucleases recognise protospacers with a minimal T-rich PAM, and their small size (700-800 residues) together with the lack of a transactivation crRNA (traccrRNA) to build the functional RNP, make Cas12j an atypical family of miniaturized RNA-guided nucleases (Supplementary Fig. 1).

Cas9 is the best-characterized member of Class 2 (Type II-A) and it has been developed into a genome editing tool ${ }^{9-15}$. Diverse Cas9 nuclease variants are involved in clinical trials to develop new therapies against human diseases ${ }^{16}$. The redesign of the CRISPR-Cas guide RNA, as well as the modification of the protein ${ }^{17,18}$, has provided a powerful method for genome editing in biomedical and biotechnological applications ${ }^{19,20}$. In some of these applications Adeno-associated viral (AAV) vectors are commonly used for gene delivery. Yet, packaging of the genes coding for CRISPR-Cas effector complexes into an AAV vector is challenging due to its limited capacity, thus leaving little space for the insertion of additional regulatory elements. Recently, Cas12j enzymes have been shown to mediate genome editing in mammalian and plant cells ${ }^{7}$ expanding our repertoire of genome manipulation tools. The small size Cas12j RNPs can improve our genome editing approaches by alleviating the packing problems in the AAV vectors used for delivery ${ }^{20}$. However, questions regarding the detailed molecular mechanism of target DNA cleavage by Cas $12 \mathrm{j}$ nucleases remain unanswered, as no structural information is available.

Here we report the structure of Cas $12 \mathrm{j} 3$ in complex with a target DNA after cleavage, revealing the key regions involved in the PAM recognition, hybrid stabilization and activation of the catalysis. Our work sheds light on the mechanism of action of the Cas12 $\mathrm{j}$ family, facilitating the redesign of CRISPR-Cas12j nucleases for genome editing.

\section{Results}

Cas $12 \mathrm{j} 3$ characterisation. We reconstituted a functional Cas12j3 -crRNA complex and determined the structure of the enzyme after severing a target dsDNA by cryo-EM (Figs. 1-2, Supplementary Figs. 2-4, Supplementary Table 1, Methods). Cas12j3 generates an overhang of 9-11-nt by cleaving at different phosphodiester bonds (Fig. 1a, Fig. 2b). A collateral effect of target DNA cleavage is the release of indiscriminate ssDNA degradation ${ }^{7}$, which is also triggered by a ssDNA complementary to the crRNA mimicking the T-strand (Fig. 1b-c) similarly to Cas $12 \mathrm{a}^{21}$. In both cases, indiscriminate cleavage is unleashed when a minimal 12-15-nt crRNA-DNA duplex is assembled. One of the cryoEM maps (map 2) suggests that the differences observed with DNA activators longer than 18-nt can be attributed to the presence of the R-loop disturbing the entrance of the unspecific ssDNA substrate in the catalytic site (Figs. 1d, 3 map2 and Supplementary Movie 1). The appearance of different cleavage products with activators of different lengths might suggest a change in the cleavage pattern. To address this question, we performed a time-course experiment using representative target DNAs (Fig. 2e). The assay suggests that the different lengths observed in the cleavage products are related to the different cutting efficiencies, as the same bands are observed at different time points for all the activators. The activity was tested in the presence of $\mathrm{Mg}^{2+}$ and other divalent cations (Supplementary Fig. 2a). The assay revealed that $\mathrm{Mg}^{2+}, \mathrm{Mn}^{2+}, \mathrm{Fe}^{2+}, \mathrm{Co}^{2+}$, and $\mathrm{Ni}^{2+}$ support Cas12j3 catalysis, generating different cleavage products. Cas $12 \mathrm{j} 3$ activity was saturated when the endonuclease/ DNA ratio was nearly equimolar, suggesting the slow dissociation of the enzyme from the PAM-proximal cleavage product, as observed in other RNA-guided nucleases ${ }^{22,23}$ (Supplementary Fig. 2b). In addition, removing the final 39 residues, not visualized in the structure, decreased Cas $12 \mathrm{j} 3$ activity (Supplementary Fig. 2c-d).

Structure of the Cas12j3/R-loop complex. Heterogeneous refinement resulted in the visualisation of several conformations of the complex. The predominant class yielded a map at a resolution of $2.7 \AA$, which was used to build the model of the Cas $12 \mathrm{j} 3$ / R-loop structure. The high flexibility observed in other predominant classes precluded model building but revealed the flexible regions and the conformational heterogeneity of the complex, which we further supported by performing a 3D variability analysis ${ }^{24}$ (Figs. 2-3, Supplementary Figs. 3-4, Supplementary Table 1, Supplementary Movie 1, Methods).

The Cas12j3/R-loop complex does not present the classical bilobal architecture observed in other type $\mathrm{V}$ effector complexes $^{25}$. The R-loop displays a $\mathrm{T}$ shape with the crRNA/ DNA hybrid and the crRNA handle forming the horizontal and vertical bars, and the protein domains wrapping around the nucleic acids (Fig. 2d-e). The handle of the crRNA is stabilized by the strictly conserved R338 which interacts with C-1 and U-18 and the neighbouring non-canonical Watson-Crick base pair interaction between G-17 and A-2 (Supplementary Fig. 6). The PAM- proximal and distal regions of the heteroduplex are recognized by the $\mathrm{N}$ - and $\mathrm{C}$-terminal regions of the protein (Fig. 2a, d, e), which are connected by a 15-residue loop (380-395). Each region comprises around half of the size of the protein and they are separated by the long handle of the crRNA on the T-shape assembly. The $\mathrm{N}$-terminal region comprises the T-strand and NT-strand PAM interacting domains (TPID, NPID) and the RNA-handle binding domain (RBD), while the C-terminal consists of the catalytic RuvC and stop (STP) domains (Fig. 2a). The RuvC domain is split into RuvC-I and RuvC-II by the presence of the STP domain, which is connected to the catalytic domain by two long bridge helices, $\mathrm{BH}-\mathrm{I}$ and $\mathrm{BH}-\mathrm{II}$. Additionally, the RuvC-II subdomain includes a characteristic insertion, which is conserved in all Cas12j except Cas12j6 (Fig. 2a, Supplementary Fig. 1). The N- and C-terminal separation seems to be also functional, as the domains involved in RNP assembly, PAM recognition and unwinding reside in the $\mathrm{N}$-terminal section, while those stabilizing the crRNA/T-strand hybrid and catalysis of the target DNA are located in the C-terminal region of 
a
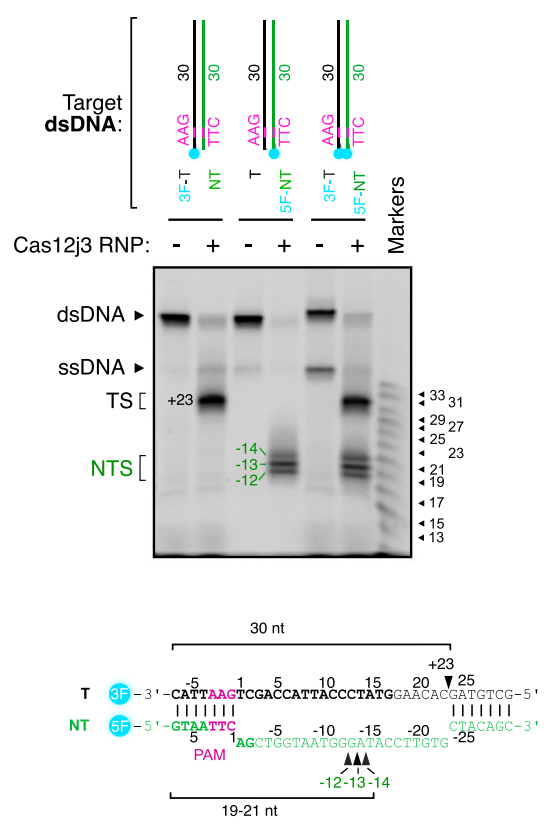

e

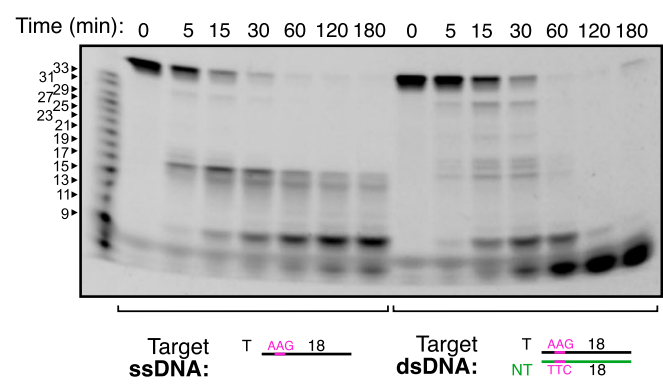

b

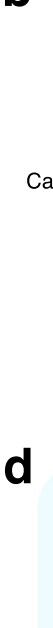

C

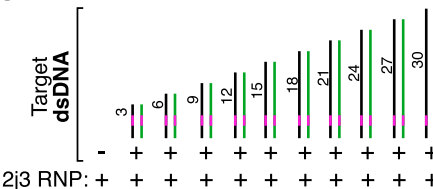

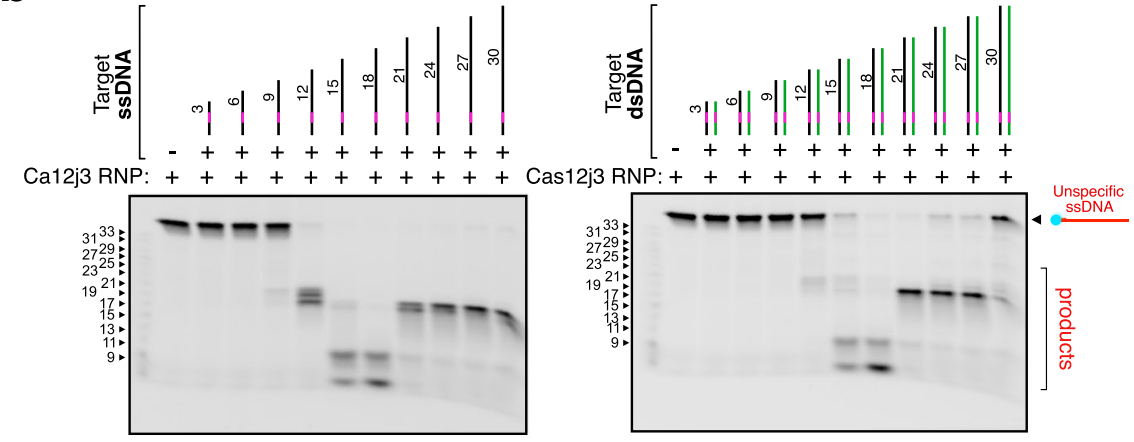
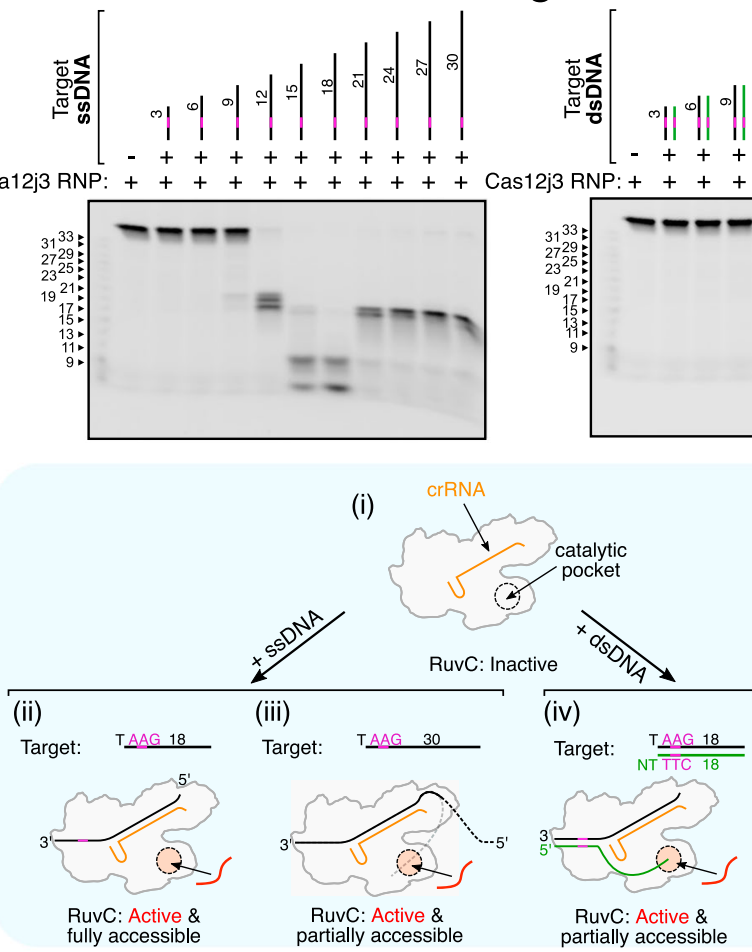

(v)

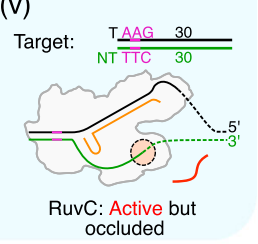

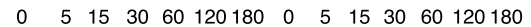

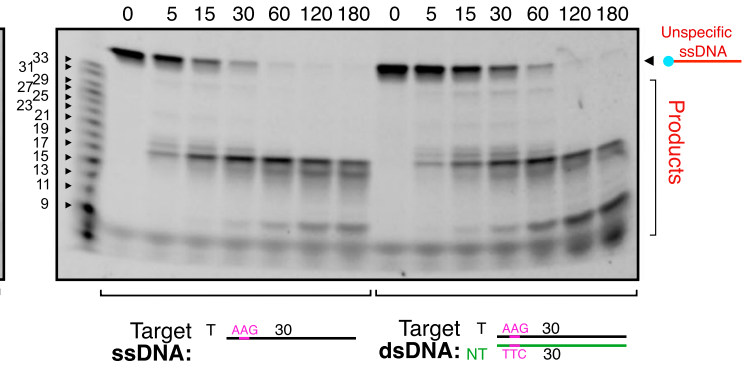

ssDNA:

Fig. 1 Cas12j3 target dsDNA cleavage and unspecific ssDNA degradation. a representative dsDNA cleavage pattern generated by Cas12j3 wild type (WT). T-strand (TS) and NT-strand (NTS) products are marked, showing a cut at positions $-13,-14$ and -15 of the NT-strand, while the T-strand is cleaved at position +23 . The sequence of the double-labelled duplex is shown below, marking the position of the cut (triangles), and the size of the labelled products. b Unspecific ssDNA degradation after activation with a specific target ssDNA of different lengths (Oligonucleotides T-AAG-3 to T-AAG-30 in Supplementary Table II). c Unspecific ssDNA degradation after activation with a specific dsDNA activator of different lengths (Oligonucleotides T-AAG-3/ NT-TTC-3 to T-AAG-30/NT-TTC-30 in Supplementary Table II). d Schematic cartoon of the results shown in b) and c). Activation of the unspecific ssDNA cleavage is observed between 12-30 nt. (i) The RuvC domain of Cas12j3 RNP is inhibited. Full activation of the unspecific cleavage is observed when using an ssDNA or dsDNA activator pairing with the crRNA between 12-18 nt (ii and iv). The use of longer oligos as ssDNA(iii) or dsDNA (v) results in a reduction of the cleavage efficiency, likely due to a steric occlusion of the catalytic site by the T-strand and NT-strand. e Unspecific ssDNA degradation time course by Cas12j3 activated by 18 and 30 nt, ss- and dsDNA targets. The DNA markers indicate the size of the products in nucleotides. The experiments are shown in $\mathbf{a}-\mathbf{c}$ and $\mathbf{e}$ are representative of three independent experiments with similar results.

the polypeptide. Therefore, the PAM site is $\sim 55 \AA$ away from the RuvC nuclease active site.

The target DNA cleavage yields a triple strand R-loop with the T-strand hybridized to the crRNA (Fig. 2b, d), while the dissociated PAM NT-strand is directed towards the catalytic pocket (Fig. 4a). The NT-strand nucleotides -1 to -2 upstream of the PAM were built in the density but the high flexibility on its distal end precluded visualization of the rest, as shown for Cas $9^{26}$ and Cas $12 \mathrm{a}^{22}$. Nevertheless, the backbone of the NT-strand is observed at a low contour level in the cryo-EM maps, suggesting the path followed by the DNA to the RuvC catalytic pocket (Supplementary Fig. 7a-b, Supplementary Movie 1). Interestingly, two nucleotides, modelled as purines, were observed in the active site in complex with $\mathrm{Ni}^{2+}$ as a by-product of the phosphodiester hydrolysis (Fig. 2c-e, Supplementary Fig. 6, Methods). To determine to which strand these nucleotides belong, we performed a binding assay after cleavage with different labelled target DNAs, revealing that these nucleotides originate from the NT-strand (Supplementary Fig. 7c-g).

PAM recognition. PAM recognition is an important aspect of DNA targeting by CRISPR-Cas nucleases, as it is a prerequisite for target DNA identification, strand separation and crRNA-targetDNA heteroduplex formation ${ }^{27}$ before cleavage. Cas $12 j 3$ recognizes a $5^{\prime}-T_{T N}-3^{\prime}$ PAM sequence in the NT-strand ${ }^{7}$. Our structure shows that PAM recognition in Cas12j3 is achieved by combining interactions in both strands by the TPID and NPID domains (Fig. 4b, 
a
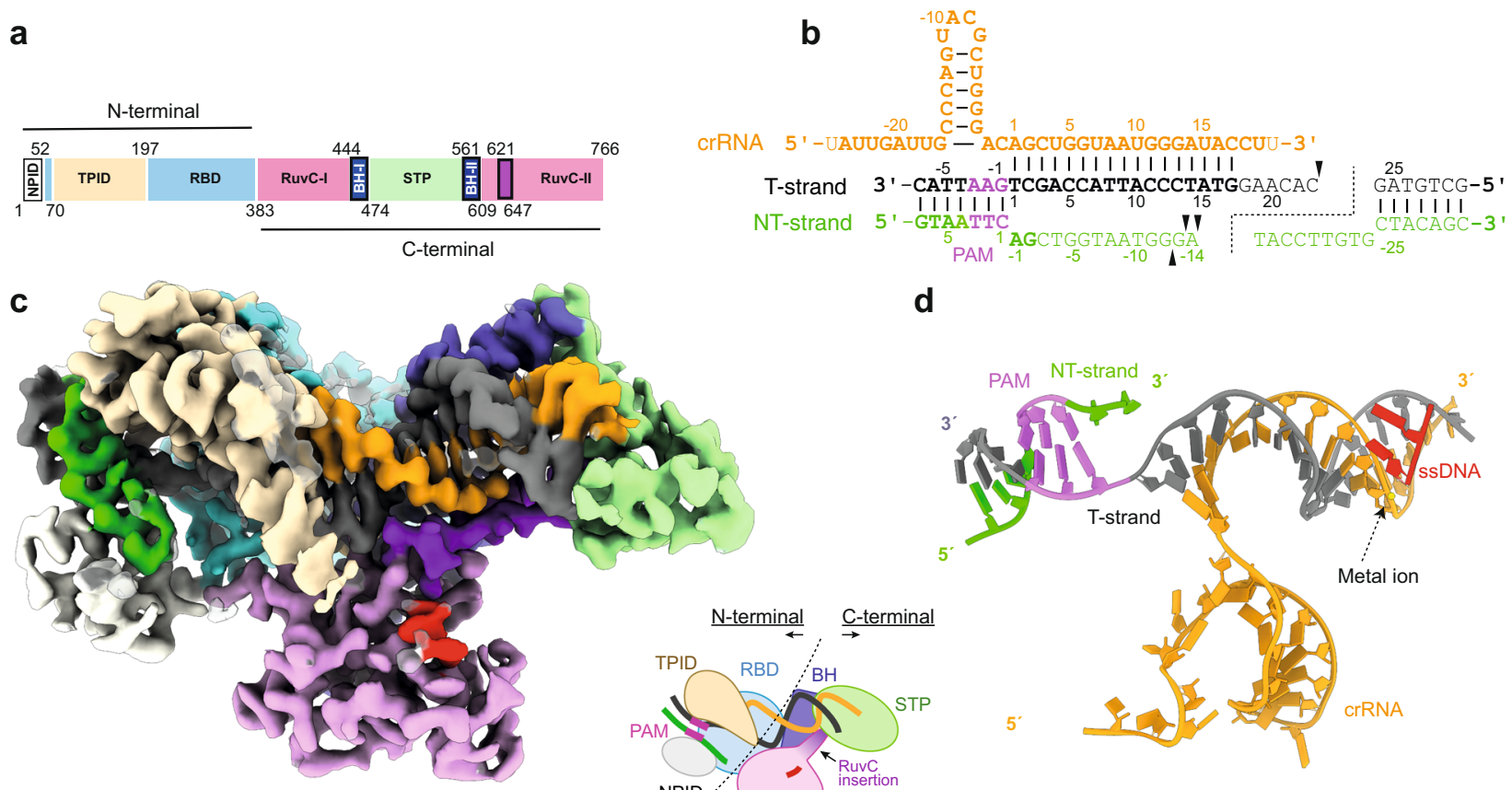

d
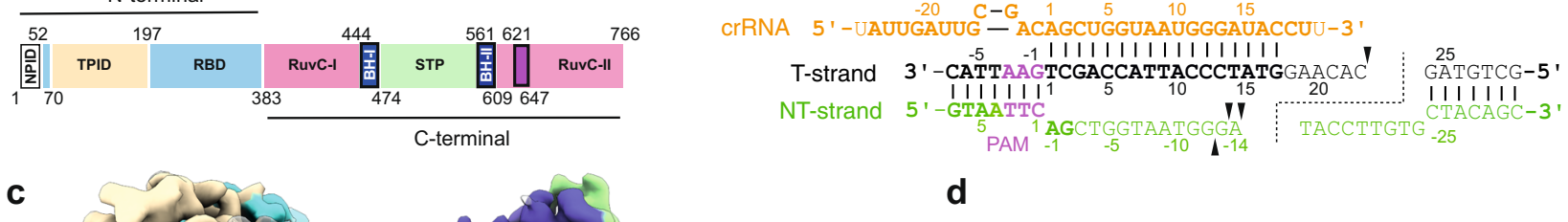

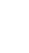
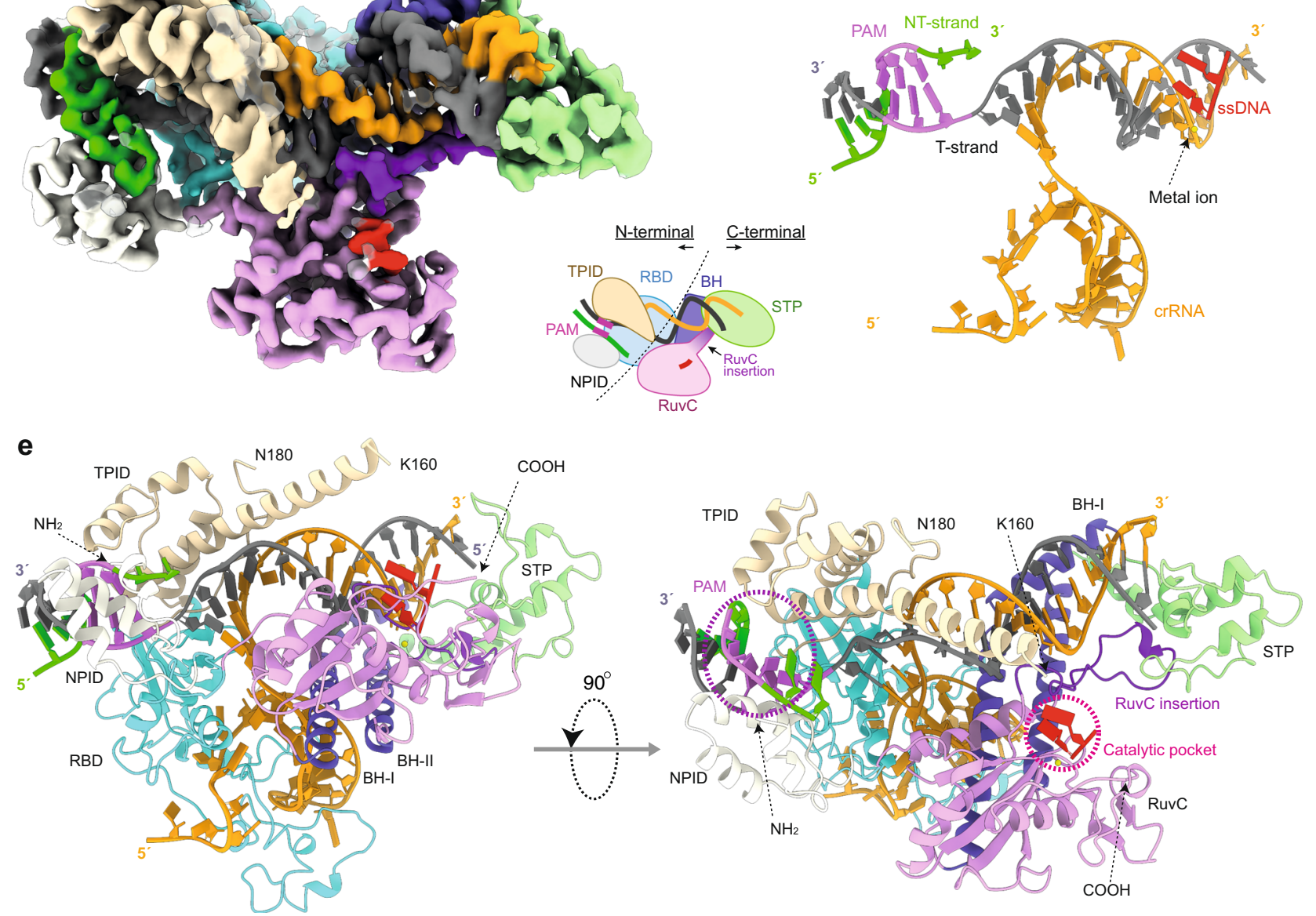

Fig. 2 Cryo-EM structure of Cas12j3 R-loop complex after target DNA cleavage. a Domain architecture of Cas12j3 comprising the T-strand and NTstrand PAM interacting domains (TPID, NPID), the RNA-handle binding domain (RBD), the bridge helices (BH-I and BH-II), the RuvC domain including the insertion and the stop (STP) domain. $\mathbf{b}$ Schematic diagram of the R-loop formed by the crRNA and the target DNA. Triangles represent phosphodiester cleavage positions in the T- and NT-strands; the bold font nucleotides represent those visualized in the structure. The PAM distal products are separated by a dashed line to illustrate the post-catalytic state (see Supplementary Fig. 7c-g). c cryo-EM map of the Cas12j3/R-loop complex at $2.7 \AA$ resolution. The cartoon depicts the relative orientation of the domains and the $\mathrm{N}$ - and $\mathrm{C}$-terminal regions of the protein. The map and the explicative cartoon are coloured according to Fig. 2a. d View of the R-loop structure and the dinucleotide in the catalytic site (polypeptide omitted). e Overview of the Cas12j3-RNA-targetDNA ternary complex (Supplementary Figs. 3-5, Supplementary Table 1).

Supplementary Fig. 5b, Supplementary Fig. 6). The positively charged side of helix a1 (S21 to A34) in the NPID is inserted in the minor groove at an angle of $45^{\circ}$ with respect to the dsDNA longitudinal axis. The conserved $\mathrm{K} 26$ and $\mathrm{K} 30$, interact with the NTstrand. K30 makes contact with $\mathrm{dT}+2$, while $\mathrm{K} 26$ is placed inside the dsDNA disrupting the Watson-Crick base coupling, displacing the NT-strand and stabilizing the separation (Fig. 4b-c, Supplementary Fig. 5b-c). On the other side of the PAM recognition cleft, Q123 in the TPID builds an intricate network of polar interactions with $\mathrm{dA}-3$ and $\mathrm{dA}-2$ in the T-strand and the $\mathrm{dT}+3$ in the NTstrand (Fig. 4b, Supplementary Fig. 6). The neighbouring G198 amide contacts the carbonyl of Q123, anchoring the side chain in a conformation favouring the contacts with these bases. In addition, the side chain of Q197 interacts with Q123 and hydrogen bonds with dA-3.

The Q123A and Q197A mutations present $~ 90 \%$ activity reduction, while the $\mathrm{K} 30 \mathrm{~A}$ mutant reduces cleavage by $\sim 55 \%$. The triple mutant activity is similar to the Q123A/Q197A mutant, indicating the pivotal role of the glutamines in PAM recognition, as the addition of the $\mathrm{K} 30 \mathrm{~A}$ mutation does not display a further reduction (Fig. 4d-e). The K26A mutant does not affect activity, suggesting that unzipping may proceed without the insertion of this residue and that the observed conformation of the helix al stabilizes the unzipped dsDNA after unwinding in the postcatalytic state. None of these mutants changed the cleavage pattern (Fig. 4d-e). However, neither wild type nor the mutants 

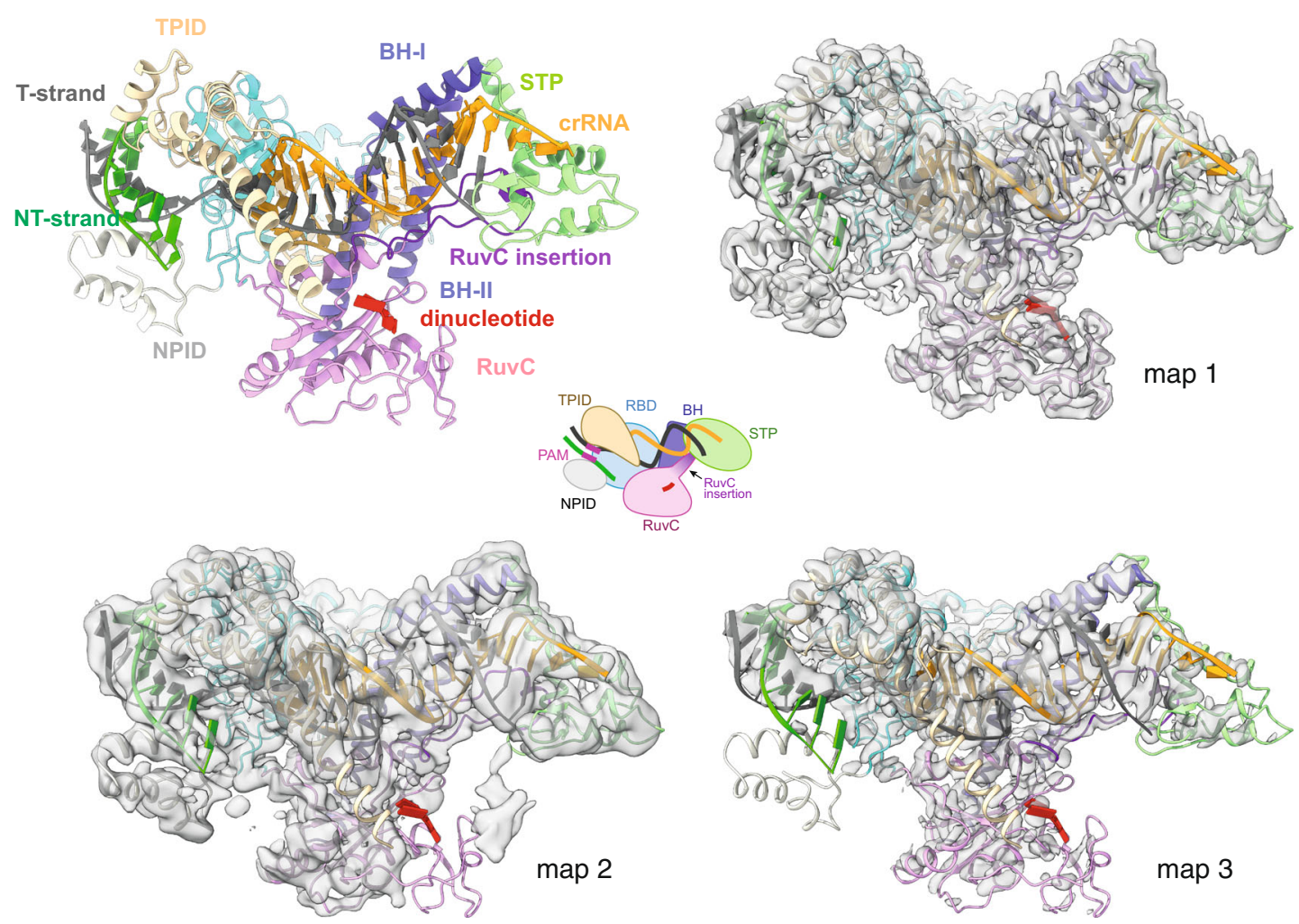

Fig. 3 Cas12j3/R-loop atomic model and cryo-EM maps. Comparison of the three cryo-EM maps (Supplementary Fig. 3d, Supplementary Fig. 4 and Supplementary Table 1). The top left ribbon diagram and the central cartoon are included to facilitate the comparison between the maps. The maps indicate the high flexibility of the NPID, RuvC and STP domains and extra density from the T-strand in map2 (Supplementary Movie 1).

cleaved target dsDNAs with different PAM sequences or in the absence of PAM, underscoring the selectivity of the PAM interaction network formed by Q123A, Q197A, and K30A (Supplementary Fig. 8a).

Next, we assessed the role of the PAM complementary bases in the T-strand by triggering the unspecific activity of Cas $12 \mathrm{j} 3$ using ssDNAs activators mimicking the T-strand with different PAM sequences (Supplementary Fig. 8b). As expected, the unspecific ssDNA catalysis is also activated in the presence of target dsDNA, suggesting that after PAM recognition crRNA/DNA hybrid assembly activates catalysis (Fig. 1c, Supplementary Fig. 8b) in a fashion similar to Cas $12 \mathrm{a}^{21}$. To assess the role of the PAM complementary bases in the T-strand, we triggered the unspecific activity of Cas12j3 using ssDNA activators with different PAM complementary sequences. The assay showed that the PAM complementary $3^{\prime}-\mathrm{AAG}-5^{\prime}$ and an activator without PAM unleashed phosphodiester hydrolysis, while other sequences promoted no or lower levels of activation, suggesting that the assembly of a hybrid duplex longer than 12-nt unleashes catalysis (Fig. 1b-c, Supplementary Fig. 8b). Activators containing regions that build shorter duplexes or partially hybridize with the crRNA display lower cleavage (Supplementary Fig. 8b).

Collectively, the combined structure-function analysis suggests that the well-conserved Q123 and Q197 residues play an essential role in PAM recognition. The direct base readout in the PAM region of Cas12j nucleases combine interactions of the TPID and NPID with both strands of the target DNA, and the interactions of the TPID with the T-strand seem to have an important role in PAM discrimination.

The unwound T-strand is stabilized by hybrid assembly. Overlaying with the PAM site, the antiparallel $\beta$-sheet consisting of the RBD $\beta 1, \beta 6$ and $\beta 7$ (Supplementary Fig. 1), builds a cavity where the dissociated T-strand is stabilized by the hybridisation with the crRNA in the post-catalytic stage (Fig. 4c). This cavity is flanked on the C-terminal region by the $\mathrm{BH}-\mathrm{I}$ helix and the RuvC domain. The well-conserved F54, K55, P56, P57, P363, T360, G361, D362, and V364 organize the cavity combining acidic and hydrophobic residues facilitating the Watson-Crick coupling of $\mathrm{dT}+1$ and $\mathrm{A}+1$ between the T-strand and the crRNA (Fig. 4c, Supplementary Fig. 5c, Supplementary Fig. 6). In addition, the backbone phosphate group of dG- 1 is recognized by the side chain of the T360, K55 and the main chain of Y376. This interaction results in the rotation of the phosphate group (Fig. 4c, Supplementary Fig. 6), thus facilitating base pairing between $\mathrm{dT}+1$ and $\mathrm{A}+1$ in the crRNA as observed in other CRISPR-Cas nucleases ${ }^{21,22,28-31}$. The neighbouring K377A mutation led to a $\sim 20 \%$ decrease, while the T360A and K55A mutations reduced the activity by 50 and $60 \%$. However, the double mutant activity is $\sim 10 \%$, highlighting the importance of these residues for hybrid formation (Fig. 4d-e).

In the post-catalytic cleavage state represented in our structure, helix $a 7$ in the TPID directs the crRNA/T-strand hybrid into the nest formed by the $\mathrm{BH}-\mathrm{I}$ and II and the RuvC insertion, detaching the hybrid from the NT-strand and preventing a possible reannealing. The conformation of $a 7$ observed in our structure complicates the initial assembly of the hybrid, as the T-strand would need to access through a narrow passage in order to anneal with the crRNA. The region of the TPID comprising a 7 seems rather flexible, as we cannot visualize 20 residues (160-180), which connect $\alpha 7$ to the rest of the TPID (Fig. 2e). This observation is also supported by the $3 \mathrm{D}$ variability analysis of our structure (Supplementary Movie 1). Therefore, we suggest that the conformation of $\alpha 7$ in the apo and pre-catalytic stages should be different to the configuration observed in our post-catalytic state structure, in order to facilitate the initial stages of unwinding and hybrid formation. 


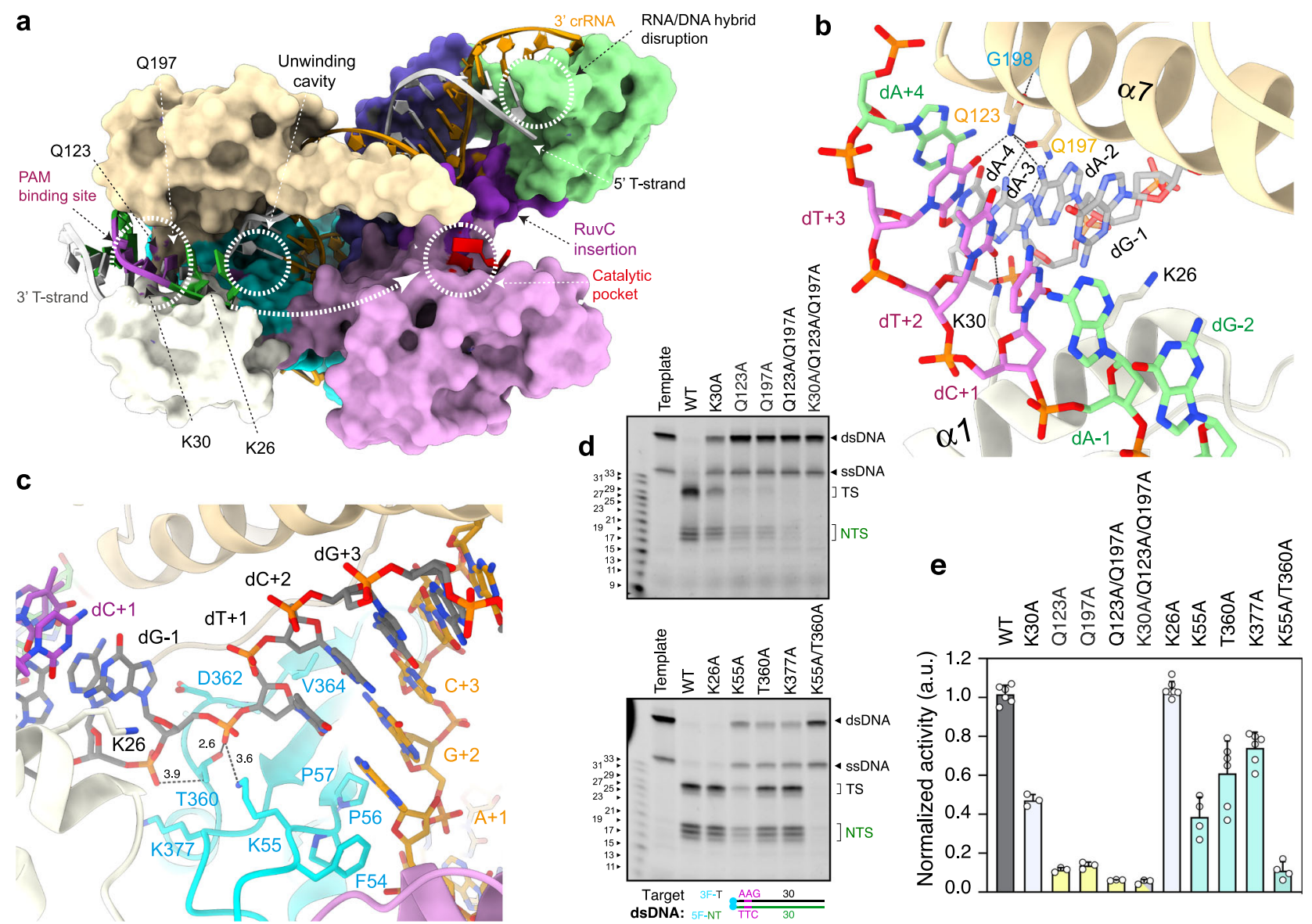

Fig. 4 Cas12j3 PAM recognition, uncoupling of the Watson-Crick dA-1:dT +1 pair and unzipping. a Surface representation of Cass12j3-R-loop complex. The white dashed arrow shows the predicted path of the NT-strand to the nuclease site after dG-2 (Supplementary Fig. 6). $\mathbf{b}$ Detailed view of the PAM nucleotides recognition and dsDNA unwinding depicting the conserved K26, K30, Q123, and Q197 residues (Supplementary Fig. 5c). c Zoom of the dT+1/ dA-1 pair uncoupling, phosphate inversion and unzipping (Supplementary Fig. 5d). d dsDNA cleavage assays using Cas12j3 wild type and PAM unwinding, activation and catalytic mutants. Oligonucleotides 3F-T-AAG-30 and 5F-NT-TTC-30 were used as substrates (Supplementary Table II). T-strand (TS) and NT-strand (NTS) products are marked. DNA markers are shown in nucleotides. e Quantification of the activity based on the cleavage experiments as shown in d. Bars represent mean \pm SD. $n>3$ independent experiments.

The area where the hybrid rests is flanked by the catalytic RuvC and STP domains, which disrupts the crRNA/T-strand hybrid as a vessel bulb bow (Fig. 5a). An antiparallel $\beta$-sheet formed by $\beta 11$ and $\beta 12$ splits the Watson-Crick base coupling after the $\mathrm{dG}+17$ : $\mathrm{C}+17$ pair; thus, limiting the hybrid length to 17 nucleotides in agreement with cleavage experiments testing the efficiency of the spacer length ${ }^{7}$. The aromatic ring of F538 in $\beta 11$ promotes hybrid unzipping (Fig. 5a), This chemical trick to disrupt the duplex is also observed in Cas12a $\mathrm{a}^{22,30,31}$. However, the arrangement of the protein moiety in Cas12a and Cas $12 \mathrm{j} 3$ is different. While in Cas12a the REC2 domain acts like the stop at the end of the train track, in Cas12j3 the STP domain splits the duplex as a blade separating the crRNA and the T-strand. The $3^{\prime}$-phosphate of the crRNA is guided to the back side of the domain where $\mathrm{C}+17$ and $\mathrm{U}+18$ are accommodated by a combination of basic (R535, R547) and hydrophobic residues (M500, L555). The 5'-phosphate of the T-strand is directed to the opposite side where the RuvC catalytic pocket is located.

Activation of catalysis. The RuvC insertion runs alongside the crRNA strand of the hybrid, making multiple contacts with its phosphate backbone from $\mathrm{U}+9$ to $\mathrm{G}+13$ (Fig. 5a, Supplementary Fig. 6, Supplementary Fig. 5d). The turn at the tip of the RuvC insertion, comprising the conserved W636, F639 and F640 hydrophobic residues, is anchored in a cleft of the STP domain, composed also by the conserved hydrophobic A490, W510, M513 residues (Fig. 5b, Supplementary Fig. 5e). This arrangement together with the activity assays (Fig. 1b-c), suggest that hybrid assembly could trigger changes in the RuvC domain transmitted through the insertion, thus activating catalysis by making the catalytic pocket available for the substrate. The monitoring of the unspecific ssDNA cleavage using activators of different lengths (Fig. 1b-c), shows that this activity of Cas $12 \mathrm{j} 3$ is fully released when the activator builds a 12-nt crRNA/DNA hybrid or longer, supporting the notion that a certain duplex length stimulates catalysis. The conserved G630 and R643 are key residues organizing a network of polar interactions with the $G+12$ phosphate (Supplementary Fig. 1), resulting in a special organisation of connections observed in our post-catalytic state structure (Fig. 5a). We hypothesize that the assembly of the hybrid and its interaction with the RuvC insertion could trigger the activity. Additionally, the conformations observed in the $3 \mathrm{D}$ variability analysis suggests positioning the STP domain towards the catalytic site facilitating the T-strand access to the active site with the proper $5^{\prime}-3^{\prime}$ polarity (Supplementary Movie 1).

We analysed substitutions in G630 and R643 that could destabilize the interaction of the hybrid with the RuvC insertion. The G630A mutation exhibited a minor activity decrease $~ 10 \%$ (Fig. $5 c-d$ ), in agreement with the G630 contribution to the polar 

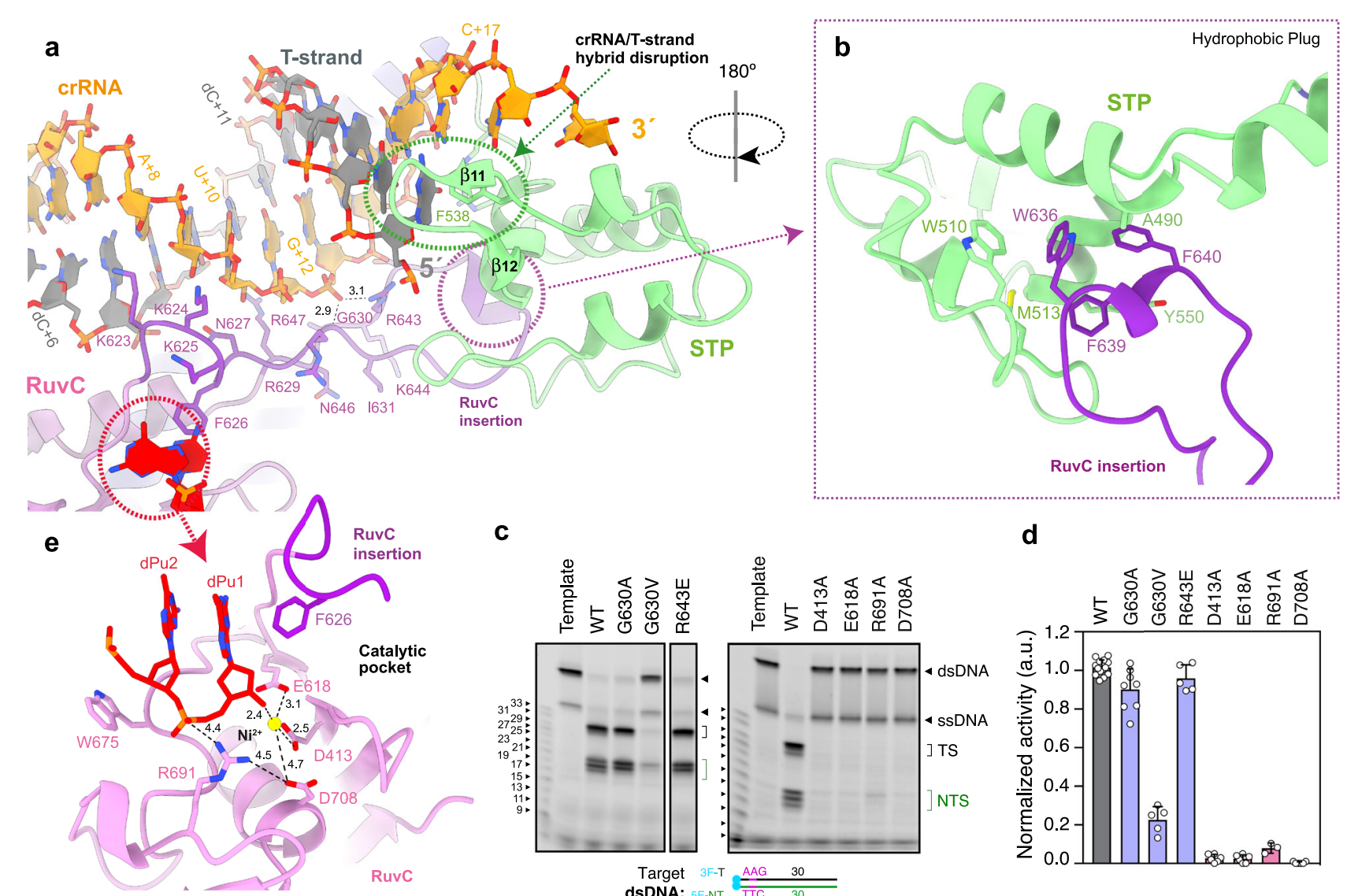

C

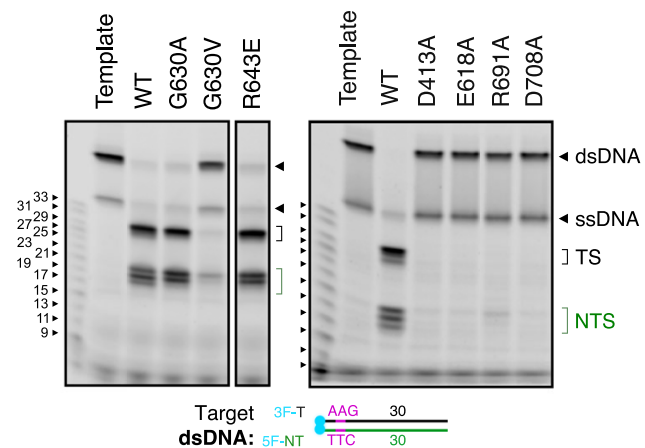

d

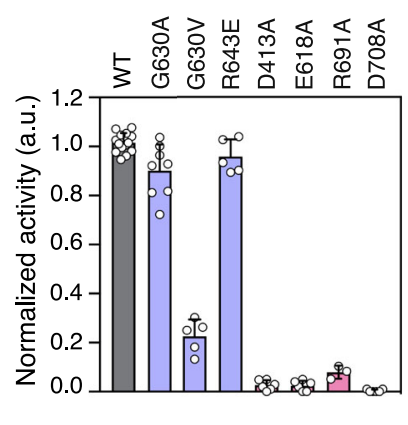

Fig. 5 Assembly of the crRNA/DNA hybrid and activation of the RuvC pocket. a View of the hybrid showing the interaction of the crRNA with residues in the RuvC insertion (Supplementary Fig. 5d). b Inset depicting the hydrophobic interaction between the turn of the RuvC insertion and the and cavity in the STP domain (Supplementary Fig. 5f). c dsDNA cleavage assays using Cas12j3 wild type and PAM unwinding, activation and catalytic mutants.

Oligonucleotides 3F-T-AAG-30 and 5F-NT-TTC-30 were used as substrates (Supplementary Table II). T-strand (TS) and NT-strand (NTS) products are marked. DNA markers are shown in nucleotides. d Quantification of the activity based on the cleavage experiments as shown in $\mathbf{c}$ Bars represent the mean \pm SD. $n>3$ independent experiments. e Detailed view of the catalytic site containing a dinucleotide and a divalent metal. The D708 side chain and the associated distances are shown for visualization purposes only (see Supplementary Fig. 5f).

network through its main chain. However, the G630V substitution displayed a strong reduction, suggesting that a bulkier side chain would destabilize the interaction with the $\mathrm{G}+12$ phosphate. Interestingly, the reversed polarity mutant R643E presented a minimal cleavage reduction of the target dsDNA (Fig. $5 \mathrm{c}-\mathrm{d}$ ), however, its indiscriminate ssDNA degradation activity showed $\sim 100 \%$ reduction, as did the G630V mutant (Supplementary Fig. 8c-d). Therefore, substitutions destabilizing the RuvC insertion can abrogate DNA cleavage and also modify the severing properties of Cas12j3, suggesting that mutations in this conserved region could be used to redesign the Cas $12 \mathrm{j}$ family of endonucleases.

In addition, all the PAM and phosphate inversion mutants, where the target DNA is not recognized and the hybrid cannot be stabilized, display indiscriminate ssDNA activity when the same assay was performed using an ssDNA activator lacking the PAM (Supplementary Fig. 8c-d). This activator would skip recognition and unwinding, thus hybridising with the complementary crRNA and triggering activation. However, when the PAM is present in the target dsDNA the variants displayed a minimal activity, as their PAM recognition and hybrid assembly are compromised, in agreement with their specific target dsDNA cleavage activity (Fig. $4 \mathrm{~d}-\mathrm{e}$ ). These results support the proposed model, as the PAM and phosphate inversion mutants would skip recognition and unwinding when activated with ssDNA, thus hybridising with the crRNA and triggering the nuclease activity.
Collectively, the data suggest that PAM recognition, DNA unwinding and activation are linked in the presence of a target dsDNA, while catalytic activation can omit PAM recognition if a suitable ssDNA is provided. Furthermore, mutations in the section of the RuvC insertion interacting with the hybrid affect the enzyme activity and change its pattern as observed in the case of the G630V and R643E mutants. Finally, substitutions in this region can also dissociate the indiscriminate ssDNA activity from the specific target dsDNA cleavage (Fig. 5c-d, Supplementary Fig. $8 c-d$ ).

Cas12j3 catalytic site. The RuvC domain of Cas12j nucleases belongs to the retroviral integrase superfamily that displays a characteristic RNaseH fold. The two nucleotides from the NTstrand in the catalytic Cas12j3 pocket are associated with the conserved E618 and D413 (Fig. 5e). The density did not allow base identification, and either $\mathrm{dA}$ or $\mathrm{dG}$ could be modelled. We built two guanines with a $5^{\prime}-3^{\prime}$ polarity and a $\mathrm{Ni}^{2+}$ ion in the density, in agreement with the number of nucleotides in the cleavage products and the purine-rich sequence in that position (Methods, Fig. 2b, Fig. 5e, Supplementary Figs. 6, 7). The length of the DNA after DSB generation could permit that the cleaved NT-strand remains associated with the catalytic centre and may disturb the entrance of the T-strand delaying its catalysis ${ }^{7}$ (Fig. 1e, Supplementary Fig. 7, Supplementary Movie 1). A second metal atom, modelled as $\mathrm{Zn}$, is coordinated by 4 conserved cysteines, similarly to Cas12 $\mathrm{g}^{32}$ and the dimeric Cas12f $\mathrm{f}^{33}$ (Fig. 6a-b). This section of 
a
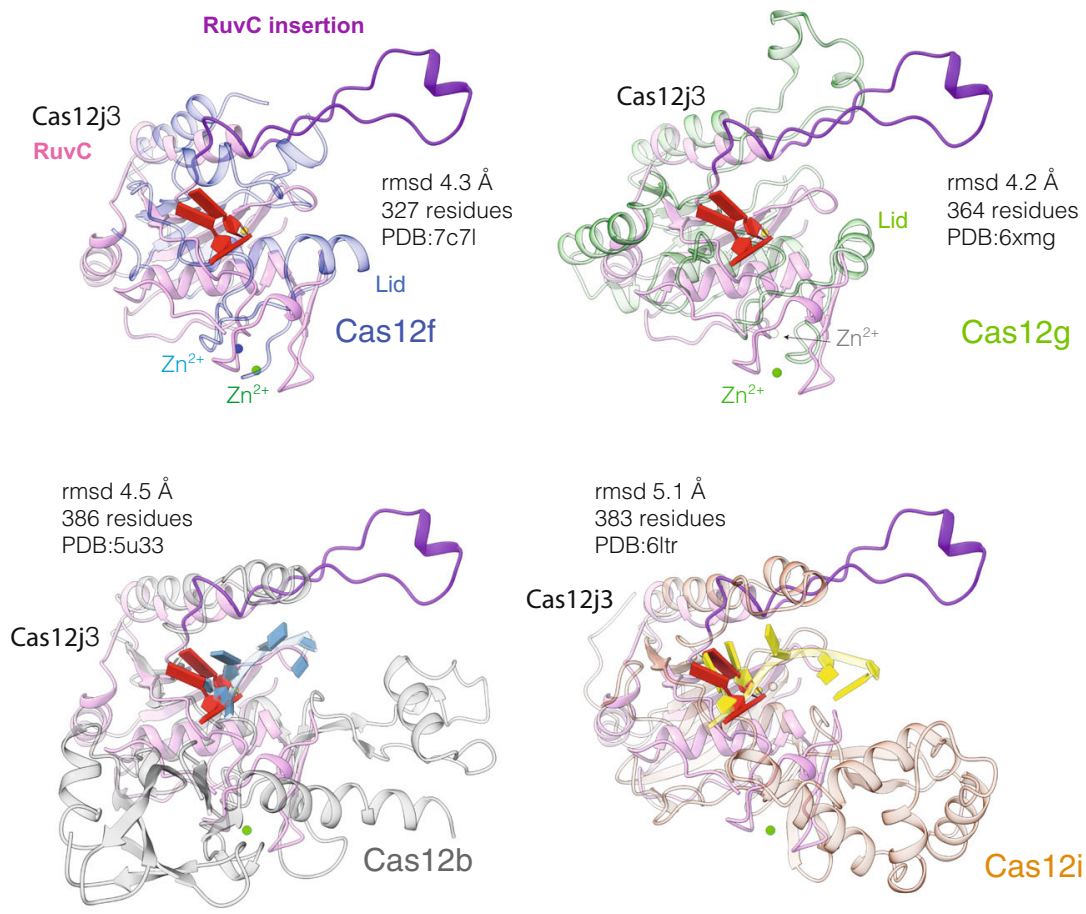

b
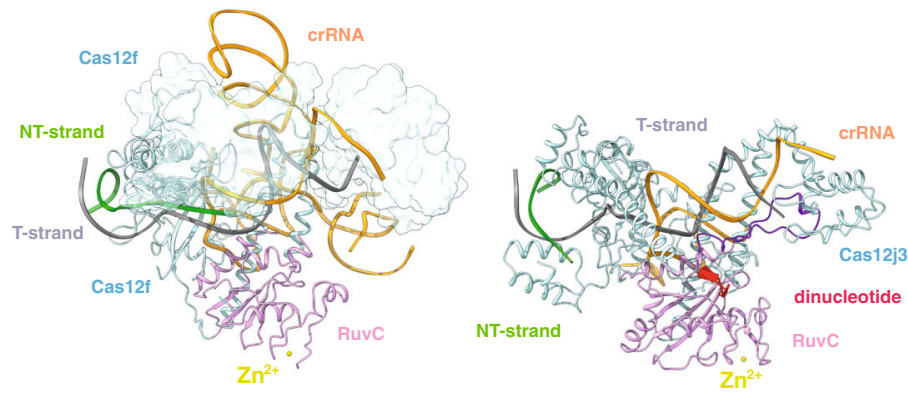

C

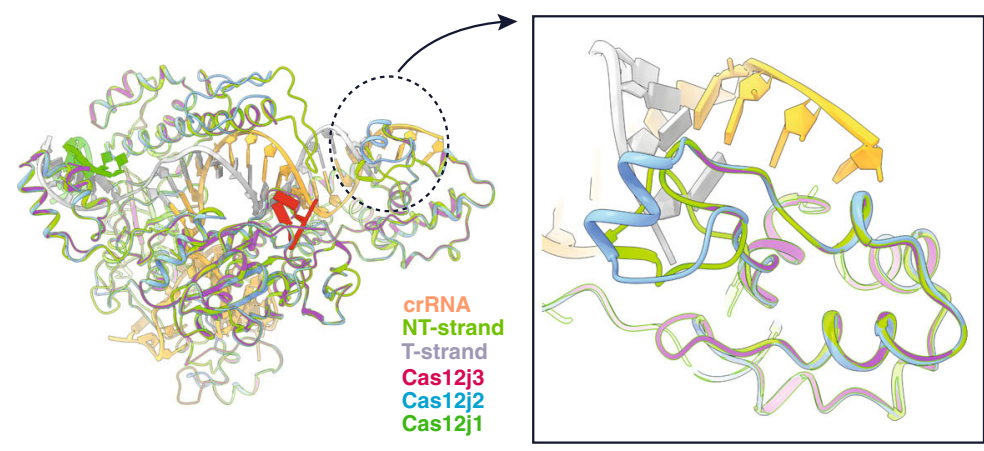

Fig. 6 Structural comparison of Cas12j3 RuvC domain with other Cas nucleases. A structural homology search of Cas12j3 against the PDB was performed using DALI ${ }^{39}$. Only the RuvC domain displays homology with other Cas nucleases. a The top panel shows the superposition of Cas $12 \mathrm{j} 3$ with Cas $12 \mathrm{f}$ and Cas12g. Both Cas12f and Cas12g present a $\mathrm{Zn}^{2+}$ atom coordinated by 4 conserved cysteines as Cas12j3. The rest of the domain is different to the TNB domain in Cas12f and Cas12g. Bottom panel Superposition of Cas12j3 with and Cas12i. Both Cas12b and Cas12i present DNA in the catalytic site and the Nuc domain inserted in the RuvC. b Detailed comparison of Cas12j3 and Cas12f after superposition in the RuvC domain. One of the monomers of the dimeric Cas12f is shown in surface representation for clarity. c Homology modelling of Cas12j1 and Cas12j using Cas12j3 and superposition of the three Cas12j family members. The inset shows the differences in the STP domain.

RuvC includes the conserved R691 $4.4 \AA$ away from the dinucleotide, whose mutation abrogates activity (Fig. 5c-d). This residue could facilitate the positioning of the phosphodiester backbone in the catalytic pocket for catalysis (Fig. 5e). However, the rest of this region is different to the target nucleic acid-binding (TNB) domain in Cas12f and Cas12g (also known as Nuc for Cas12a and Cas12b and the target-strand loading domain for
Cas12e), as it displays a different structure without the helical regulatory lid motif (Fig. 6a).

RuvC domains introduce $5^{\prime}$-phosphorylated cuts and involve three acidic amino acids ${ }^{34}$ and two divalent metal ions ${ }^{35}$. The E618 and D413 carboxylates are important catalytic residues, and their mutations abolish Cas12j3 activity (Fig. 5c-d). Both are predicted to coordinate the metal ions that activate the 
nucleophile and stabilize the transition state and the leaving group. In our structure, E618 and D413 coordinate the metal and the backbone of the dinucleotide (Fig. 5e, Supplementary Fig. 6). The side chain of D708, which is predicted to act as the third catalytic residue, is not observed due to electron irradiation ${ }^{36}$. This residue has been shown less critical than the other carboxylates in other RuvC domains, as its substitutions to Asn or His lead to a partial loss of cleavage ${ }^{37,38}$. However, in the case of Cas12j3 the D708A mutation abrogates activity (Fig. $5 \mathrm{c}-\mathrm{d}$ ). Structural comparisons using DALI with other RuvC domains support a two-metal ion mechanism (Fig. 6a, Supplementary Table 3).

Taking advantage of the high similarity between Cas12j1, Cas12j2 and Cas12j3 we performed homology modelling using our structure as a template (Methods). The superposition of the models reveals features in the STP domain supporting the different T-strand cleavage kinetics ${ }^{7}$ (Figs. 6c, 1e, Methods). The different insertions in the STP domain observed in Cas12j1 and Cas12j2 may facilitate positioning of the T-strand for cleavage. Although homology is high within the Cas $12 \mathrm{j}$ family, we did not observe differences between the Cas12j1 and Cas12j2 models with our structure that could explain why Cas $12 \mathrm{j} 3$ is unable to process its own crRNA, (Fig. 6c, Supplementary Fig. 1).

\section{Discussion}

Genome editing has radically altered life sciences, as the possibility to modify a chosen DNA sequence has triggered a race to find and develop tools useful to conduct a precise and safe modification of a genome. In the past decade, CRISPR-Cas nucleases have been harnessed to target different DNA sequences, triggering a genome editing revolution with stark implications for biomedical and biotechnological applications. However, the large size of these nucleases generates a problem in some of their applications, as the packaging of the current CRISPR-Cas effector complexes into an AAV vector is a limiting factor. In this study, we present the first structural insight of the miniaturized CRISPR-Cas12j family. Due to their reduced size, this group of recently identified compact RNA-guided endonucleases have the potential to alleviate the delivery problems.

Cas12j nucleases display sequence homology only in their RuvC domain with other Class2 types V members ${ }^{7}$. A detailed DALI ${ }^{39}$ search to find structural homologues and compare Cas12j3 with other proteins did not find any homology beyond the RuvC domain (Fig. 6, Supplementary Table 3). In addition, a BLAST search of the whole database for related sequences using the RuvC region (621-647 aa) did not find any homology, suggesting that this feature is only found in members of the Cas12j family. As the Cas enzyme with the shortest protein sequence described to date is Cas12f, we performed a superposition of Cas12j3 with Cas12f using their RuvC domains (Fig. 6a-b, Supplementary Table 3). The Cas12f protein is only 500 residues long; however, the functional RNP consist of a large RNA and two molecules of Cas $12 \mathrm{f}^{33}$. The Cas12f RNP complex is substantially different to Cas $12 \mathrm{j} 3$ (Fig. 6b), suggesting that besides the similarities in the catalytic site their working mechanisms should be different.

In the absence of structural information of the Cas12j3-RNA complex, we can only speculate that the RNP must be in an open conformation in the initial stage of the reaction, in order to allow unwinding of the target DNA. However, the flexibility observed in our cryoEM maps (Fig. 4, Supplementary Movie 1), suggests that after Cas12j3 binds the target DNA (Fig. 7) the PAM scanning would foster the stabilisation of NPID on the NT-strand and the interaction with the TPID would allow PAM binding. Our structure provides us with a snapshot of the R-loop stabilized after cleavage. In this conformation, the hybrid is formed and separated from the NT-strand by the helix $\alpha 7$. We hypothesize that $\alpha 7$ should be in a different conformation to permit dsDNA unwinding and it would

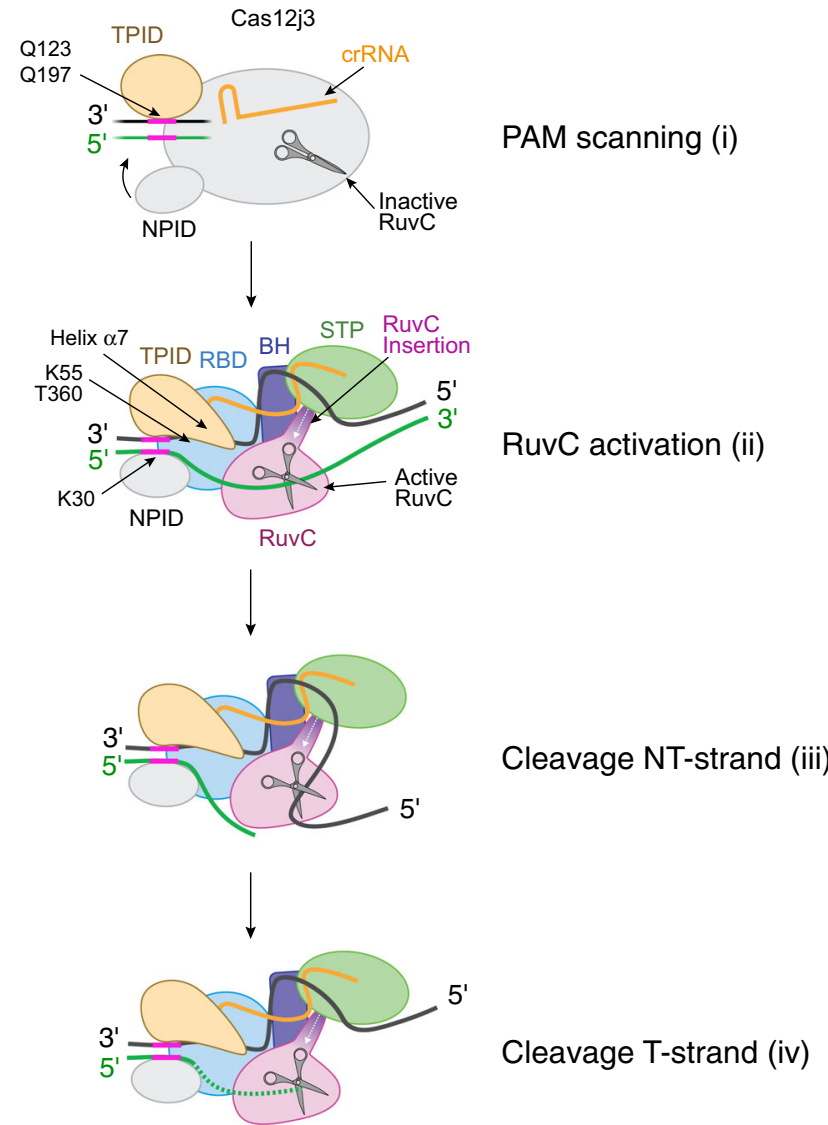

Fig. 7 Model of Cas12j3 PAM-dependent DNA recognition, unwinding and cleavage. Cartoon model depicting the stages of Cas12j3 nuclease staggered target DNA cleavage (see Discussion). The T-strand and NT-strand are illustrated in green and black, with the PAM colored in pink. In step (i) the $\mathrm{RBD}, \mathrm{BH}, \mathrm{STP}$, and RuvC domains are represented as an oval (see Discussion).

clip the hybrid after it has been stabilized by the phosphate inversion. The NT-strand would be directed towards the catalytic site (Fig. 7, Supplementary Movie 1, Supplementary Fig. 7), while the STP domain disrupts the hybrid and orients the T-strand towards the RuvC pocket (Fig. 3 map 2). We propose that the length of the hybrid would be monitored by the RuvC insertion, promoting the activity of the enzyme if the length of the duplex is longer than 12nt. Catalysis would proceed on the NT-strand, which will access the catalytic pocket with the $5^{\prime}-3$ polarity and later on the T-strand, generating the overhang on the dsDNA. The unspecific ssDNA cleavage activity would be triggered by a similar mechanism after crRNA/target DNA assembly.

Our work provides molecular detail of Cas $12 j 3$ PAM recognition, hybrid stabilisation and activation mechanisms. This study paves the way for the rational redesign of these compact RNPs, widening the CRISPR-Cas toolbox. The repurposing of these miniaturized RNA-guided nucleases will open new avenues for their use in genome editing biomedical applications, where the size of the current CRISPR-Cas systems is a challenge.

\section{Methods}

Plasmid preparation, protein expression and purification. Cas $12 \mathrm{j} 3 \mathrm{cDNA}$ was synthetized and cloned with a C-terminal hexahistidine (His)-tag into pET-21 vector (Genewiz) (Supplementary Table 2). Cas12j3 mutants were generated with the InFusion cloning kit (Takara) using the primers specified in Supplementary Table II. To generate Cas12j3- $\triangle \mathrm{CT}$, a TEV cleavage site (ENLYFQG) was generated after the residue M726. His-tagged Cas12j3 was expressed from pET-21 in E. coli BL21 pRARE cells. E. coli cultures were grown at $37^{\circ} \mathrm{C}$ in a liquid Terrific Broth (TB) medium with $34 \mathrm{mg} / \mathrm{l}$ chloramphenicol and $100 \mathrm{mg} / \mathrm{l}$ ampicillin to an optical density at $600 \mathrm{~nm}$ of $\sim 0.8$. Both 
the wild type and mutants were expressed and purified following the same protocol. Only mutations in the insertion turn (W636A, F639A, and F640A) and the STP cleft (W510A, M513A) rendered Cas12j3 insoluble, highlighting the importance of this conserved interaction in the Cas12j family. Overexpression of proteins was induced with $150 \mathrm{nM}$ of IPTG for $16 \mathrm{~h}$ at $16^{\circ} \mathrm{C}$. Cells were harvested by centrifugation and resuspended in lysis buffer (50 mM HEPES pH7.5, $2 \mathrm{M} \mathrm{NaCl}, 5 \mathrm{mM} \mathrm{MgCl} 2,1$ tablet of Complete Inhibitor cocktail EDTA Free (Roche) per $50 \mathrm{ml}, 50 \mathrm{U} / \mathrm{ml}$ Benzonase, $1 \mathrm{mg} /$ $\mathrm{ml}$ lysozyme). Lysis was completed by one freeze-thaw cycle and sonication. Cell extract was diluted to a final salt concentration of $500 \mathrm{mM}$, and high-speed centrifuged $(10,000 \times \mathrm{g}, 45 \mathrm{~min})$ to separate the soluble fraction from the insoluble fraction and the cell debris. The soluble fraction was loaded into a $5 \mathrm{ml}$ HisTrap FF Crude column (Cytiva) equilibrated in buffer IMAC-A (20 mM HEPES pH7.5, $500 \mathrm{mM} \mathrm{NaCl}, 20 \mathrm{mM}$ Imidazole), and bound proteins were eluted by stepwise increase of the imidazole concentration with buffer IMAC-B (20 mM HEPES pH7.5, $200 \mathrm{mM} \mathrm{KCl,} 500 \mathrm{mM}$ Imidazole). Cas12j3 proteins eluted at $\sim 150 \mathrm{mM}$ Imidazole. In the case of Cas12j3$\Delta \mathrm{CT}$, the C-terminal segment (residues 727-766) was cleaved by incubating the protein with $0.3 \mathrm{mg}$ TEV protease in TEV buffer (20 mM HEPES pH 7.5, $150 \mathrm{mM} \mathrm{NaCl}, 1 \mathrm{mM}$ EDTA, $0.5 \mathrm{mM}$ TCEP) for $16 \mathrm{~h}$ at $4{ }^{\circ} \mathrm{C}$. Fractions containing Cas12j3 were pooled, concentrated and further purified by size exclusion chromatography (SEC) using a HiLoad 16/600 Superdex 200 column (Cytiva) equilibrated in SEC buffer ( $20 \mathrm{mM}$ HEPES pH7.5, $500 \mathrm{mM} \mathrm{KCl}, 0.5 \mathrm{mM}$ TCEP). Fractions containing pure protein were pooled, concentrated to $5-10 \mathrm{~g} / \mathrm{L}$, flash-frozen in liquid nitrogen and stored at $-80^{\circ} \mathrm{C}$.

Cleavage assays. Fluorescein (FAM)-labeled DNA oligonucleotide at $5^{\prime}$ or $3^{\prime}$ ends, unlabeled DNA and RNA oligonucleotides were purchased from Integrated DNA Technologies (IDT) (Supplementary Table II). dsDNA substrates were prepared by mixing ssDNA oligos to a final concentration of $80 \mu \mathrm{M}$ in annealing buffer $(20 \mathrm{mM}$ HEPES pH7.5, $200 \mathrm{mM} \mathrm{KCl}$ ), denaturation at $95^{\circ} \mathrm{C}$ for $10 \mathrm{~min}$ and gradually temperature decrease to $4^{\circ} \mathrm{C}$ during $20 \mathrm{~min}$ in a thermal cycler (Applied Biosystems). Ribonucleoprotein complexes (RNP) of Cas12j3 were formed by mixing an equal volume of $50 \mu \mathrm{M}$ Cas $12 j 3$ and $50 \mu \mathrm{M}$ Cas $12 j 3$ mature crRNA (IDT).

For specific dsDNA cleavage assays, FAM-labeled dsDNA substrates were incubated at $400 \mathrm{nM}$ with $2 \mu \mathrm{M}$ of Cas12j3 RNP in cleavage buffer (20 mM HEPES pH7.5, $160 \mathrm{mM} \mathrm{KCl}, 10 \%$ glycerol, $5 \mathrm{mM} \mathrm{MgCl} 2$ ) for $2 \mathrm{~h}$ at $37^{\circ} \mathrm{C}$, or as otherwise stated in the figure legends. For ion dependency assays (Supplementary Fig. 2a) $5 \mathrm{mM}$ $\mathrm{MgCl}_{2}$ was substituted by $5 \mathrm{mM}$ Ethylenediaminetetraacetic acid (EDTA), $\mathrm{CaCl}_{2}$ $\mathrm{MnCl}_{2}, \mathrm{FeSO}_{4}, \mathrm{CoCl}_{2}, \mathrm{NiSO}_{4}, \mathrm{CuCl}_{2}, \mathrm{ZnSO}_{4}$. For DNA saturation experiments (Supplementary Fig. 2b) $1 \mu \mathrm{M}$ of Cas12j3 RNP was incubated with $0.5-8 \mathrm{uM}$ of labelled dsDNA for $2 \mathrm{~h}$ at $37^{\circ} \mathrm{C}$. For unspecific trans ssDNA cleavage assays (Fig. 1b-c, Supplementary Fig. 8b-c), $0.4 \mu \mathrm{M}$ FAM-labeled unspecific ssDNA substrate (i.e., not complementary to the crRNA) was incubated with $2 \mu \mathrm{M}$ Cas12j3 RNP as described above, along with $0.1 \mu \mathrm{M}$ of unlabeled activator ssDNA or dsDNA (complementary to the crRNA) in cleavage buffer for $1 \mathrm{~h}$ at $37^{\circ} \mathrm{C}$. The reactions were stopped by adding equal volumes of stop buffer ( $8 \mathrm{M}$ Urea, $100 \mathrm{mM}$ EDTA at pH8) followed by incubation at $95^{\circ} \mathrm{C}$ for $5 \mathrm{~min}$. Cleavage products were resolved on $15 \%$ Novex TBE-Urea Gels (Invitrogen), run according to the manufacturer's instructions. Gels were imaged using an Odyssey FC Imaging System (Li-Cor). Densitometric analysis of bands in gels was performed using ImageJ. The cleavage efficiency was calculated as the intensity of the bands corresponding to the products divided by the total intensity for the specific dsDNA cleavage assays, or as the depletion of a signal of the noncleaved product for unspecific ssDNA degradation assays.

Sample preparation for Cryo-EM. For the preparation of the Cryo-EM sample, $\mathrm{Ni}^{2+}$ was used as a catalytic ion instead of $\mathrm{Mg}^{2+}$ due to the higher yield obtained with this metal. Cas12j3 RNP was prepared as described above (cleavage assays). $25 \mathrm{nmol}$ of RNP and $37 \mathrm{nmol}$ of unlabeled dsDNA substrate were incubated in $25 \mathrm{ml}$ of MonoQ A buffer $\left(20 \mathrm{mM}\right.$ HEPES pH7.5, $200 \mathrm{mM} \mathrm{KCl}, 1 \mathrm{mM} \mathrm{NiSO}_{4}$, $0.5 \mathrm{mM}$ TCEP) for $2 \mathrm{~h}$ at $20^{\circ} \mathrm{C}$ to allow DNA cleavage. The product of the reaction was loaded in a MonoQ column equilibrated with MonoQ A buffer, and Cas12j3/R-loop complex was separated from the RNP and the unbound DNA substrate by a salt gradient elution using MonoQ B buffer (20 mM HEPES pH7.5, $2 \mathrm{M} \mathrm{KCl}, 1 \mathrm{mM} \mathrm{NiSO}_{4}, 0.5 \mathrm{mM}$ TCEP). Cas12j3/R-loop eluted at $16-20 \%$ of MonoQ buffer B $(\sim 500 \mathrm{mM} \mathrm{KCl})$. The R-loop complex was further purified from unbound DNA by SEC using a Superdex 200 Increase 10/300 GL column (Cytiva) equilibrated with MonoQ A buffer. The molecular weight of the complex and the sample homogeneity was estimated using a Refeyn One mass photometer (Refeyn), using 10-20 nM of protein diluted in MonoQ A buffer (Supplementary Fig. 3a). 2.5 $\mu \mathrm{L}$ of freshly purified Cas12j3/R-loop complex (Absorbance $260 \mathrm{~nm}$ of $\sim 1.6$ ) was applied to UltrAuFoil 300 mesh R0.6/1.0 holey grids (Quantifoil), glow-discharged for $60 \mathrm{~s}$ at $10 \mathrm{~mA}$ (Leica EM ACE200), and plunge-frozen in liquid ethane (pre-cooled with liquid nitrogen) using a Vitrobot Mark IV (FEI, Thermo Fisher Scientific) using the next conditions: blotting time $3 \mathrm{~s}, 100 \%$ humidity and $4{ }^{\circ} \mathrm{C}$.

CryoEM data collection and processing. Movies were collected on Titan Krios G3 Cryo-TEM equipped with a TFS Falcon III camera operated at $300 \mathrm{keV}$ in counting mode. Exposure $1.05 \mathrm{e} / \AA^{2} /$ frame, in 40 frames and hence a final dose of $42 \mathrm{e} / \AA^{2}$. The calibrated pixel size was $0.832 \AA / \mathrm{px}$. All movies were pre-processed using WARP
1.0.940 (Supplementary Fig. 3). Motion correction was performed with a temporal resolution of 20 for the global motion and $5 \times 5$ spatial resolution for the local motion. We considered motion in the $45-3 \AA$ range weighted with a B-factor of $-500 \AA^{2}$. Only Micrographs displaying less than $5 \AA$ intraframe motion were used. CTF estimation was performed using $5 \times 5$ patches in the $35-4 \AA$ range. We selected micrographs with fitted defocus between 0.0 and $5.0 \mu \mathrm{m}$, and a resolution better than $5 \AA$ For the particle picking, the micrographs were masked, and particles were picked using a re-trained BoxNet deep convolutional neural network. This resulted in $3,504,102$ particles from 4393 micrographs. Particles were extracted with a box size of $256 \times 256$ and a pixel size of 0.832 which were inverted and normalized before being imported into RELION $3.1^{41}$ for $2 \mathrm{D}$ classification. The selected 2D classes were imported in cryoSPARC $3.1 .0^{42}$ where they were $3 \mathrm{D}$ classified into four initial classes. The volume with the largest number of particles was refined to an initial $2.61 \AA$ resolution map. The conformational heterogeneity of the particles used in this volume was inspected using the 3D variability analysis in cryoSPARC ${ }^{24,42}$. This method allows the resolution and visualization of the molecular motions taking place in the observed particles. The two more divergent volumes obtained in the $3 \mathrm{D}$ variability analysis were used as input for heterogeneous refinement and subsequent nonuniform refinement jobs, yielding maps at 2.6 and $2.8 \AA$ resolution. We further analysed the heterogeneity of the particles used to reconstruct these two maps by performing $3 \mathrm{D}$ variability analysis/heterogeneous refinement/non-uniform refinement jobs, resulting in 6 maps at 2.7-3.3 resolution (Supplementary Fig. 3d). The three best maps represent the three conformations of the complex that are discussed in the text (depicted as map 1, map 2, and map 3 Supplementary Table 1). Sharpened and local resolution maps were calculated with PHENIX ${ }^{43}$, and directional resolution anisotropy analysis was performed with the 3D-FSC server ${ }^{44}$.

Atomic model building and refinement. An initial model containing the complete DNA and RNA sequence and $\sim 50 \%$ of the protein sequence was built ab initio using map-to-model implemented in PHENIX ${ }^{43}$. COOT ${ }^{45}$ was used to connect, extend and correct the protein fragments to generate a model covering $\sim 70 \%$ of the protein sequence. The rest of the model was auto built by using a buccaneer implemented in CCP-EM ${ }^{46}$ and subsequently corrected in COOT. The final model was obtained after several rounds of refinement using Phenix.real_space_refine and manual inspection and correction in COOT. The final model covers $92 \%$ of the protein sequence, mainly lacking a C-terminal segment predicted to be unstructured. Map and molecular model images were created using Chimera $\mathrm{X}^{47}$. The complete polypeptide and R-loop complex were built in the high-resolution map. Besides the flexible $\mathrm{N}$ - and C-terminal the only gap in the protein sequence is the loop between residues 160-180. The extra density in the catalytic pocket allowed us to identify the presence of two nucleotides. The density allowed the modelling of $\mathrm{dA}$ or $\mathrm{dG}$ in the density. We built and a couple of $\mathrm{dG}$ nucleotides, in agreement with the abundance of these nucleotides in the NT-strand at that position (Fig. 2b)

Structural homology models. The high sequence identity between Cas12j1, 2 and $3(\sim 40 \%$, Supplementary Fig. 1$)$ and the good coverage of the sequence by our structural model allows the generation of structural homology models for Cas12j1 and Cas12j2 with relatively high confidence. These models were generated by using the SWISS-MODEL server (https://swissmodel.expasy.org) using our Cas12j3 structure as template ${ }^{48}$. The results are displayed in (Fig. $6 \mathrm{c}$ ).

Superposition of Cas12j3 RuvC domain with other Cas12 enzymes. A DALI ${ }^{39}$ search against the complete PDB did not found homologues of Cas12j3. Only the Cas12j3 RuvC displays homology with the same domain from other Cas12 enzymes (Fig. 6a-b). The Cas12b RuvC domain is the most similar to Cas12j3 (rmsd $4.5 \AA$ for 386 residues), followed by Cas12f and Cas12g (rmsd 4.3 and $4.2 \AA$ for 364 and 327 residues respectively) and Cas12i (rmd $5.1 \AA$ for 383 residues). Cas12 endonucleases generally cleave the T-strand and NT-strand at the single RuvC active site, with the Nuc domain facilitating the loading of the NT- and T-strands into the active site. A BLAST search $^{49}$ of Cas12j3 RuvC insertion (621-647) did not find any similarity in the database, supporting this unique feature of the Cas12j family of endonucleases. Although the region of the protein facilitating the loading of the NT- and T-strand is different to other Cas 12 enzymes, the Cas $12 \mathrm{j}$ family displays 4 strictly conserved Cys residues that bind a metal, which we have modelled as $\mathrm{Zn}$ as in the case of Cas $12 \mathrm{f}$ and Cas12g (Fig. 6a). DALI performed the superposition with Cas12f in the better ordered $\mathrm{RuvC}$, as Casl2f is a dimer. This is the smallest polypeptide found in the CRISPR-Cas family. However, this $\sim 500$ residues protein dimerizes upon binding of a large crRNA, which includes a section of the tracr-RNA. Only one of the RuvC domains seems to cleave target $\mathrm{DNA}^{33}$.

Reporting summary. Further information on research design is available in the Nature Research Reporting Summary linked to this article.

\section{Data availability}

The atomic coordinates and cryo-EM maps have been deposited in the Protein Data Bank and EMDB under accession codes 7ODF and EMD-12827. Source data are provided with this paper. All other data are available from the corresponding author upon request. Source data are provided with this paper. 
Received: 20 June 2021; Accepted: 5 July 2021;

Published online: 22 July 2021

\section{References}

1. Abudayyeh, O. O. et al. C2c2 is a single-component programmable RNAguided RNA-targeting CRISPR effector. Science 353, aaf5573 (2016).

2. Horvath, P. \& Barrangou, R. CRISPR/Cas, the immune system of bacteria and archaea. Science 327, 167-170 (2010).

3. Sorek, R., Lawrence, C. M. \& Wiedenheft, B. CRISPR-mediated adaptive immune systems in bacteria and archaea. Annu Rev. Biochem 82, 237-266 (2013).

4. Gasiunas, G., Barrangou, R., Horvath, P. \& Siksnys, V. Cas9-crRNA ribonucleoprotein complex mediates specific DNA cleavage for adaptive immunity in bacteria. Proc. Natl Acad. Sci. 109, 2579-2586 (2012).

5. Yan, W. X. et al. Cas13d is a compact RNA-targeting type VI CRISPR effector positively modulated by a WYL-domain-containing accessory protein. Mol. Cell 70, 327-339 e325 (2018).

6. Al-Shayeb, B. et al. Clades of huge phages from across Earth's ecosystems. Nature 578, 425-431 (2020).

7. Pausch, P. et al. CRISPR-CasPhi from huge phages is a hypercompact genome editor. Science 369, 333-337 (2020).

8. Chen, J. S. et al. CRISPR-Cas12a target binding unleashes indiscriminate single-stranded DNase activity. Science 360, 436-439 (2018).

9. Jinek, M. et al. A programmable dual-RNA-guided DNA endonuclease in adaptive bacterial immunity. Science 337, 816 (2012).

10. Cong, L. et al. Multiplex genome engineering using CRISPR/Cas systems. Science 339, 819-823 (2013).

11. Jinek, M. et al. RNA-programmed genome editing in human cells. eLife 2, e00471 (2013).

12. Kleinstiver, B. P. et al. Genome-wide specificities of CRISPR-Cas Cpf1 nucleases in human cells. Nat. Biotechnol. 34, 869-874 (2016).

13. Kim, D. et al. Genome-wide analysis reveals specificities of Cpf1 endonucleases in human cells. Nat. Biotechnol. 34, 863-868 (2016).

14. Zetsche, B. et al. Cpf1 is a single RNA-guided endonuclease of a class 2 CRISPR-Cas system. Cell 163, 759-771 (2015).

15. Hsu, P. D., Lander, E. S. \& Zhang, F. Development and applications of CRISPR-Cas9 for genome engineering. Cell 157, 1262-1278 (2014).

16. Ernst, M. P. T. et al. Ready for repair? Gene editing enters the clinic for the treatment of human disease. Mol. Ther. Methods Clin. Dev. 18, 532-557 (2020).

17. Cox, D. B. T. et al. RNA editing with CRISPR-Cas13. Science 358, 1019-1027 (2017).

18. Walton, R. T., Christie, K. A., Whittaker, M. N. \& Kleinstiver, B. P. Unconstrained genome targeting with near-PAMless engineered CRISPRCas9 variants. Science 368, 290-296 (2020).

19. Barrangou, R. \& Horvath, P. A decade of discovery: CRISPR functions and applications. Nat. Microbiol 2, 17092 (2017).

20. Saha, K. et al. The NIH somatic cell genome editing program. Nature 592, 195-204 (2021).

21. Stella, S. et al. Conformational activation promotes CRISPR-Cas12a catalysis and resetting of the endonuclease activity. Cell 175, 1856-1871 e1821 (2018).

22. Stella, S., Alcon, P. \& Montoya, G. Structure of the Cpf1 endonuclease R-loop complex after target DNA cleavage. Nature 546, 559-563 (2017).

23. Sternberg, S. H., Redding, S., Jinek, M., Greene, E. C. \& Doudna, J. A. DNA interrogation by the CRISPR RNA-guided endonuclease Cas9. Nature 507, 62-67 (2014).

24. Punjani, A. \& Fleet, D. J. 3D variability analysis: Resolving continuous flexibility and discrete heterogeneity from single particle cryo-EM. J. Struct. Biol. 213, 107702 (2021).

25. Stella, S., Alcon, P. \& Montoya, G. Class 2 CRISPR-Cas RNA-guided endonucleases: Swiss army knives of genome editing. Nat. Struct. Mol. Biol. 24, 882-892 (2017).

26. Jiang, F. et al. Structures of a CRISPR-Cas9 R-loop complex primed for DNA cleavage. Science 351, 867-871 (2016).

27. Anders, C., Niewoehner, O., Duerst, A. \& Jinek, M. Structural basis of PAMdependent target DNA recognition by the Cas9 endonuclease. Nature 513, 569-573 (2014).

28. Jiang, F., Zhou, K., Ma, L., Gressel, S. \& Doudna, J. A. STRUCTURAL BIOLOGY. A Cas9-guide RNA complex preorganized for target DNA recognition. Science 348, 1477-1481 (2015).

29. Swarts, D. C. \& Jinek, M. Mechanistic insights into the cis- and trans-acting DNase activities of Cas12a. Mol. Cell 73, 589-600 (2019)

30. Swarts, D. C., van der Oost, J. \& Jinek, M. Structural basis for guide RNA processing and seed-dependent DNA targeting by CRISPR-Cas12a. Mol. Cell 66, 221-233 e224 (2017).

31. Yamano, T. et al. Crystal structure of Cpf1 in complex with guide RNA and target DNA. Cell 165, 949-962 (2016).
32. Li, Z., Zhang, H., Xiao, R., Han, R. \& Chang, L. Cryo-EM structure of the RNA-guided ribonuclease Cas12g. Nat. Chem. Biol. 17, 387-393 (2021).

33. Takeda, S. N. et al. Structure of the miniature type V-F CRISPR-Cas effector enzyme. Mol. Cell 81, 558-570.e553 (2021).

34. Nowotny, M. Retroviral integrase superfamily: the structural perspective. EMBO Rep. 10, 144-151 (2009).

35. Steitz, T. A. \& Steitz, J. A. A general two-metal-ion mechanism for catalytic RNA. Proc. Natl Acad. Sci. USA 90, 6498-6502 (1993).

36. Bartesaghi, A., Matthies, D., Banerjee, S., Merk, A. \& Subramaniam, S. Structure of $\beta$-galactosidase at 3.2- $\AA$ resolution obtained by cryo-electron microscopy. Proc. Natl Acad. Sci. 111, 11709 (2014).

37. Chapados, B. R. et al. Structural biochemistry of a type 2 RNase H: RNA primer recognition and removal during DNA replication. J. Mol. Biol. 307, 541-556 (2001).

38. Kanaya S. Enzymatic activity and protein stability of E. coli ribonuclease H. In: Crouch, R. J. \& Toulmé, J. J. (eds), Ribonucleases H. John Libbey, Paris, pp. $1-38,(1998)$.

39. Holm, L. \& Rosenström, P. Dali server: conservation mapping in 3D. Nucleic Acids Res. 38, W545-W549 (2010).

40. Tegunov, D. \& Cramer, P. Real-time cryo-electron microscopy data preprocessing with Warp. Nat. Methods 16, 1146-1152 (2019).

41. Zivanov, J. et al. New tools for automated high-resolution cryo-EM structure determination in RELION-3. Elife 7, https://doi.org/10.7554/eLife.42166 (2018).

42. Punjani, A., Rubinstein, J. L., Fleet, D. J. \& Brubaker, M. A. cryoSPARC algorithms for rapid unsupervised cryo-EM structure determination. Nat. Methods 14, 290-296 (2017).

43. Liebschner, D. et al. Macromolecular structure determination using X-rays, neutrons and electrons: recent developments in Phenix. Acta Crystallogr. D. 75, 861-877 (2019).

44. Tan, Y. Z. et al. Addressing preferred specimen orientation in single-particle cryo-EM through tilting. Nat. Methods 14, 793-796 (2017).

45. Emsley, P. \& Cowtan, K. Coot: model-building tools for molecular graphics. Acta Crystallogr. D. 60, 2126-2132 (2004).

46. Burnley, T., Palmer, C. M. \& Winn, M. Recent developments in the CCP-EM software suite. Acta Crystallogr. Sect. D. 73, 469-477 (2017).

47. Goddard, T. D. et al. UCSF ChimeraX: Meeting modern challenges in visualization and analysis. Protein Sci. 27, 14-25 (2018).

48. Guex, N. \& Peitsch, M. C. SWISS-MODEL and the Swiss-PdbViewer: an environment for comparative protein modeling. Electrophoresis 18, 2714-2723 (1997).

49. Altschul, S. F., Gish, W., Miller, W., Myers, E. W. \& Lipman, D. J. Basic local alignment search tool. J. Mol. Biol. 215, 403-410 (1990).

\section{Acknowledgements}

We thank the Danish Cryo-EM National Facility in CFIM at the University of Copen hagen for support during cryo-EM data collection supported by grant NNF0024386. Data processing has been performed at the Computerome, the Danish National Computer for Life Sciences. G.M. is a member of the Integrative Structural Biology Cluster (ISBUC) at the University of Copenhagen. SE is funded by the Novo Nordisk Foundation (Grant NNF17OC0030788). G.M. is part of the Novo Nordisk Foundation Center for Protein Research (CPR), which is supported financially by the Novo Nordisk Foundation (grant NNF14CC0001). This work was also supported by the cryoNET (grant NNF17SA0030214), and Distinguished Investigator (NNF18OC0055061) grants to G.M.

\section{Author contributions}

A.C., A.F., S.S., and G.M. designed the biochemical experiments. A.C., A.F., and P.T. set up the purification protocol. A.C. created the mutants and performed biochemistry experiments using Cas $12 \mathrm{j} 3$ mutants. P.T. purified Cas $12 \mathrm{j} 3 \mathrm{WT}$ and mutants. A.F. performed most of the biochemistry analysis. A.C., A.F., and G.M. analysed the biochemical data. A.C. and A.F. prepared the cryo-EM sample, EM grids and collected the cryo-EM images. T.P. and N.S. helped with sample preparation, data collection and advised in the initial stages of processing. SE helped with the Warp pipeline, and A.C. performed the rest of the Cryo-EM processing and built the structure. A.C., A.F., and G. M. proceeded with cryo-EM map and structure analysis. The global results were discussed and evaluated with all authors. G.M. coordinated and supervised the project and wrote the manuscript with input from all the authors.

\section{Competing interests}

Guillermo Montoya and Stefano Stella declare that they are co-founders of Twelve Bio. A patent application has been filed relating to this work. Arturo Carabias, Anders Fuglsang, Stefano Stella and Guillermo Montoya are co-inventors on the patent. The other authors declare no competing interests. 


\section{Additional information}

Supplementary information The online version contains supplementary material available at https://doi.org/10.1038/s41467-021-24707-3.

Correspondence and requests for materials should be addressed to G.M.

Peer review information Nature Communications thanks the anonymous reviewer(s) for their contribution to the peer review of this work.

Reprints and permission information is available at http://www.nature.com/reprints

Publisher's note Springer Nature remains neutral with regard to jurisdictional claims in published maps and institutional affiliations. (c) (1) Open Access This article is licensed under a Creative Commons Attribution 4.0 International License, which permits use, sharing, adaptation, distribution and reproduction in any medium or format, as long as you give appropriate credit to the original author(s) and the source, provide a link to the Creative Commons license, and indicate if changes were made. The images or other third party material in this article are included in the article's Creative Commons license, unless indicated otherwise in a credit line to the material. If material is not included in the article's Creative Commons license and your intended use is not permitted by statutory regulation or exceeds the permitted use, you will need to obtain permission directly from the copyright holder. To view a copy of this license, visit http://creativecommons.org/ licenses/by/4.0/.

(C) The Author(s) 2021 